\title{
The effects of inhaled corticosteroid on insulin sensitivity in asthmatic patients
}

\author{
Seyed Hamid Borsi1,2, Homeira Rashidi1, Maryam Shabanpoor³, Hanieh Raji2,3 \\ 1 Health Research Institute, Diabetes Research Center, Ahvaz Jundishapur University of Medical Sciences, Ahvaz \\ 2 Air pollution and Respiratory Diseases Research Center, Ahvaz Jundishapur University of Medical Sciences, Ahvaz \\ 3 Department of Internal Medicine, Faculty of Medicine, Ahvaz Jundishapur University of Medical Sciences, \\ Ahvaz, Iran
}

\begin{abstract}
Asthma is an inflammatory disease, which causes airflow limitation and increase insulin resistance. The present study was carried out in order to investigate insulin resistance and the effect of inhaled corticosteroid (ICS) on insulin sensitivity in asthmatic patients. A registered (IRCT201605247411N2) interventional, quasi-experimental trial was performed from 2014 to 2015 in Imam Khomeini hospital Ahvaz, Iran. Patients with mild to moderate asthma participated in this study. Spirometry, fasting blood sugar (FBS), blood sugar 2 hour post prandial (BS2HPP), HbA1C, low density lipoprotein (LDL), high density lipoprotein (HDL), Insulin Level, and C reactive protein (CRP) were measured. Then Homeostatic Model Assessment-Insulin Resistance [HOMA-IR] Index, and calculated. Data were analyzed using paired $t$-test, wilcoxon and McNemar's test using SPSS 20.0 Software. The study consisted of
\end{abstract}

Corresponding Author: Hanieh Raji, Air Pollution and Respiratory Diseases Research Center, Ahvaz Jundishapur University of Medical Sciences, Ahvaz, Iran. Tel/Fax +98.16.32216104. E-mail: dr.raji.h@gmail.com

Key words: Asthma; insulin resistance; inhaled corticosteroid.

Contributions: SHB, contribution to data collection, interpretation, analyses of the data and manuscript drafting; HRashidi, contribution to design of study, analyses and interpretation of data, MS involved in data collection, analyses and interpretation of data; HRaji, study design, contribution to data collection, analyses, interpretation of the data and manuscript drafting. All authors read and approved the final manuscript.

Conflict of interest: The authors declare no competing interests.

Acknowledgments: The trial protocol was approved by the institutional ethical review board at Ahvaz Jundishapur University of Medical Sciences (AJUMS). The trial was registered at Iranian Registry of Clinical Trials with registration number (IRCT201605247411N2). The cooperation of all participants in this study is greatly appreciated.

Received for publication: 6 November 2017

Accepted for publication: 28 January 2018

(C) Copyright S.H. Borsi al., 2018

Tipografia PI-ME Editrice, Italy

Monaldi Archives for Chest Disease 2018; 88:892

doi: 10.4081/monaldi.2018.892

This article is distributed under the terms of the Creative Commons Attribution Noncommercial License (by-nc 4.0) which permits any noncommercial use, distribution, and reproduction in any medium, provided the original author(s) and source are credited.
35 non-diabetic patients suffering from asthma (20 men and 15 women) with a mean age of $36.6 \pm 12.3$ years. Inhaled corticosteroid had a significant effect on spirometric parameters, but it had no significant effect on other variables. At baseline, mean HbA1C, insulin level and HOMA-IR were 5.5\%, $10.9 \mathrm{mIU} / \mathrm{l}$ and 2.7 respectively. None of these values changed significantly after treatment with inhaled corticosteroid for two months. The results indicated that there is no relationship between ICS and increased insulin resistance in asthmatic patients.

\section{Introduction}

Asthma is an inflammatory disease, which causes airflow limitation [1]. Similar to other inflammatory diseases, asthma can lead to insulin resistance. Diseases like asthma, chronic obstructive pulmonary diseases (COPD), pulmonary fibrosis and pneumonia are more prevalent among diabetic patients [2,3]. Acute intermittent hypoxia can affect glucose metabolism and reduces insulin sensitivity. For these patients, if asthma is not well-controlled, low insulin sensitivity and other disorder are likely to occur [4]. In a study about the effects of treatment on insulin sensitivity level of asthmatic patients before and after treatment the results did not statistically change; but for people who had COPD the level of glucose had a statistically remarkable increase after treatment. In addition, there was a positive relationship between forced expiratory volume 1 (FEV1) percent-change and HOMA-IR change in asthmatic patients as a result of the treatment [5]. Also in another study the effects of inhaled corticosteroid on glucose control in diabetic adults along with asthma and COPD were investigated. Measuring HbAlC 6 weeks after the treatment indicated that there was a marginal but significant difference between the two groups; however, they did not observe a remarkable difference compared to the baseline values [6]. On the other hand, insulin resistance such as obesity can be associated with an increased risk of developing asthmalike symptoms. Furthermore obesity and asthma may be stimulated inflammatory pathways and involved in insulin resistance [7].

Inhaled corticosteroids are the mainstay of treatment in asthmatic patients and commonly used. Since oral corticosteroids cause impaired glucose tolerance. The question of the relation between inhaled corticosteroid use and the increase risk of diabetes has been rekindled recently. There are different studies with contradictory results on the effects of inhaled corticosteroid on glucose control in diabetic and nondiabetic individuals $[2,5,8]$. The present study was carried out in order to investigate the status of glucose tolerance in asthmatic patients and the effects of inhaled corticosteroid in this regard.

\section{Materials and Methods}

We performed a quasi-experimental trial in Imam Khomeini hospital, Ahvaz that was conducted from 2014 to 2015. The trial protocol 
was approved by the institutional ethical review board at Ahvaz Jundishapur University of Medical Sciences (AJUMS). The trial was registered at Iranian Registry of Clinical Trials with registration number (IRCT201605247411N2). Thirty-five non-diabetics recently diagnosed asthmatic patients who were referred to pulmonary clinic, participated in this study. A pulmonologist diagnosed each patient based on the symptoms and spirometry test according to GINA 2014 guidelines.

Inclusion criteria included: age over 15 years, stage 1 or 2 asthma, FEV1 above $60 \%$, no previous use of oral and /or inhaled corticosteroid. Exclusion criteria: history of diabetes or malignity, pregnancy, acute infection, endocrine disorders, taking any medication that affects glucose metabolism, obesity (defined by $\mathrm{BMI} \geq 30 \mathrm{~kg} / \mathrm{m}^{2}$ ) and sleep apnea.

Primary outcome was HOMA-IR, 24-variable homeostasis model assessment (iHOMA2) that extends the HOMA2 model, before and after medication consumption and secondary outcomes included FEV1, FVC, FEV1/FVC, FBS, BS2HPP, HbA1C, LDL, HDL, Insulin Level and CRP. Patients' HOMA-IR was calculated and was classified as HOMA-IR $<3$ normal, 3-5 moderate and $>5$ sever. Then patients received treatment with inhaled Budesonide with a dosage of $320 \mu \mathrm{g}$ every 12 hours, for two months. Patients were asked not to change their regiment, physical activity, lifestyle and habits. There was no restriction for utilizing leukotriene antagonists, inhaled anticholinergic, and short- and longacting inhaled beta- 2 agonists during the study. At the end of intervention, all the examinations were repeated.

\section{Statistical analysis}

Kolmogorov-Smirnov test was employed to determine whether sample data are normally distributed (p-value $>0.05$ ). Data analysis was performed using descriptive statistics such as frequency, frequency percentage, quantitative and qualitative data were analyzed with paired $t$-test, Wilcoxon test, and McNemar's respectively using
SPSS 20.0 Software. We used logistic regression for analysis relation of HOMA-IR and other variables. P-value more than 0.05 was considered significant.

\section{Results}

Thirty-five asthmatic non-diabetic patients ( 20 men and 15 women) with mean age of $36.6 \pm 12.3$ years were selected. Baseline characteristics of patients are listed in (Table 1). The mean of HOMA-IR was 2.70. HOMA-IR was less than 3 in 23 patients (65.7\%), between 3 and 5 in 9 patients (25.7\%), and over 5 in 3 patients (8.6\%). CRP was negative in $71.4 \%$ of patients in the base line.

Thirty-three patients were followed until the study was finished. Changes in clinical outcomes before and after treatment in these patients are listed in (Tables 2-5). At 2 months, inhaled corticosteroid treat-

Table 1. Baseline characters of patients.

\begin{tabular}{lc} 
Variables & Mean $( \pm$ SD) \\
Age (years) & $36.6 \pm 12.3$ \\
\hline FEV1 (\%) & $62.6 \pm 9.3$ \\
\hline FVC (\%) & $69.5 \pm 10.2$ \\
\hline FEVl/FVC & $70.5 \pm 9.8$ \\
\hline FBS (mg/dl) & $101.3 \pm 10.8$ \\
\hline BS2hpp (mg/dl) & $115.6 \pm 25.7$ \\
\hline LDL (mg/dl) & $106.1 \pm 27.9$ \\
\hline HDL (mg/dl) & $46.3 \pm 9.8$ \\
\hline HbAlC (\%) & $5.5 \pm 0.4$ \\
\hline Insulin level (mIU/l) & $11.4 \pm 5.3$ \\
\hline FEVl: forced expiratory volume in 1 second; FVC: forced vital capacity; FBS: fasting blood sugar; \\
BS2hpp: blood sugar 2 hours post prandial; LDL: low-density lipoprotein; HDL: high-density \\
lipoproteins; HbAlC: hemoglobin AlC.
\end{tabular}

Table 2. Clinical characters before and after treatment.

\begin{tabular}{|c|c|c|c|}
\hline Variables & $\begin{array}{c}\text { Before treatment } \\
\text { mean }( \pm \text { SD) }\end{array}$ & $\begin{array}{c}\text { After treatment } \\
\text { mean }( \pm \text { SD) }\end{array}$ & p value \\
\hline FEV1 (\%) & $62.6( \pm 9.1)$ & $80.1( \pm 11.1)$ & $<0.001^{*}$ \\
\hline FVC (\%) & $69.5( \pm 10.2)$ & $84.2( \pm 9.1)$ & $<0.001^{*}$ \\
\hline FEV1/FVC & $70.5( \pm 9.8)$ & $82.8( \pm 10.2)$ & $<0.001^{*}$ \\
\hline FBS (mg/dl) & $101.3( \pm 10.8)$ & $100.8( \pm 11.8)$ & $0.451^{*}$ \\
\hline BS2hpp (mg/dl) & $115.5( \pm 25.7)$ & $113.9( \pm 29.0)$ & $0.535^{*}$ \\
\hline LDL (mg/dl) & $106.1( \pm 27.9)$ & $108.3( \pm 28.5)$ & $0.803^{*}$ \\
\hline HDL (mg/dl) & $46.3( \pm 9.8)$ & $46.5( \pm 10.1)$ & $0.635^{*}$ \\
\hline $\mathrm{HbAlC}(\%)$ & $5.5( \pm 0.44)$ & $5.6( \pm 0.59)$ & $0.678^{*}$ \\
\hline Insulin level (mIU/l) & $11.4( \pm 5.3)$ & $11.7( \pm 5.4)$ & $0.322^{*}$ \\
\hline HOMA-IR & $2.7( \pm 1.2)$ & $2.9( \pm 1.5)$ & $0.539 *$ \\
\hline
\end{tabular}

FEVI: forced expiratory volume in 1 second; FVC: forced vital capacity; FBS: fasting blood sugar; BS2hpp: blood sugar 2 hours post prandial; LDL: low-density lipoprotein; HDL: high-density lipoproteins; HbAlC: hemoglobin $\mathrm{Al}$; $*$-test.

Table 3. HOMA-IR frequency before and after the treatment.

\begin{tabular}{lcc} 
HOMA-IR & Before treatment & After treatment \\
$\begin{array}{l}\text { Frequency } \\
<3\end{array}$ & $23(65.7 \%)$ & $21(63.6 \%)$ \\
\hline $3-5$ & $9(25.7 \%)$ & $10(30.3 \%)$ \\
\hline$>5$ & $3(8.6 \%)$ & $2(6.1 \%)$ \\
\hline Total & $35(100 \%)$ & $33(100 \%)$ \\
\hline
\end{tabular}


ment in asthmatic patients, had no statistically significant influence on the FBS, BS2hpp, LDL, HDL, HBAlc, insulin level (Figure 1), HOMA-IR, iHOMA, Beta cell function and insulin sensitivity. No significant difference in the CRP was found at baseline or 2 months after treatment. Also the results of IHOMA, Beta cell function, and insulin sensitivity were without any change according to BMI (Table 6). Patient's compliance

Table 4. The mean of iHOMA and insulin sensitivity before and after intervention.

\begin{tabular}{lcc} 
& Mean \pm SD & p-value \\
iHOMA2B & $1.46 \pm 0.60$ & $0.673^{*}$ \\
\hline iHOMA2A & $1.54 \pm 0.70$ & \\
\hline Sensitivity B\% & $83.20 \pm 46.25$ & $0.539 *$ \\
\hline Sensitivity A\% & $75.78 \pm 31.51$ & \\
\hline *Wilcoxon test. & &
\end{tabular}
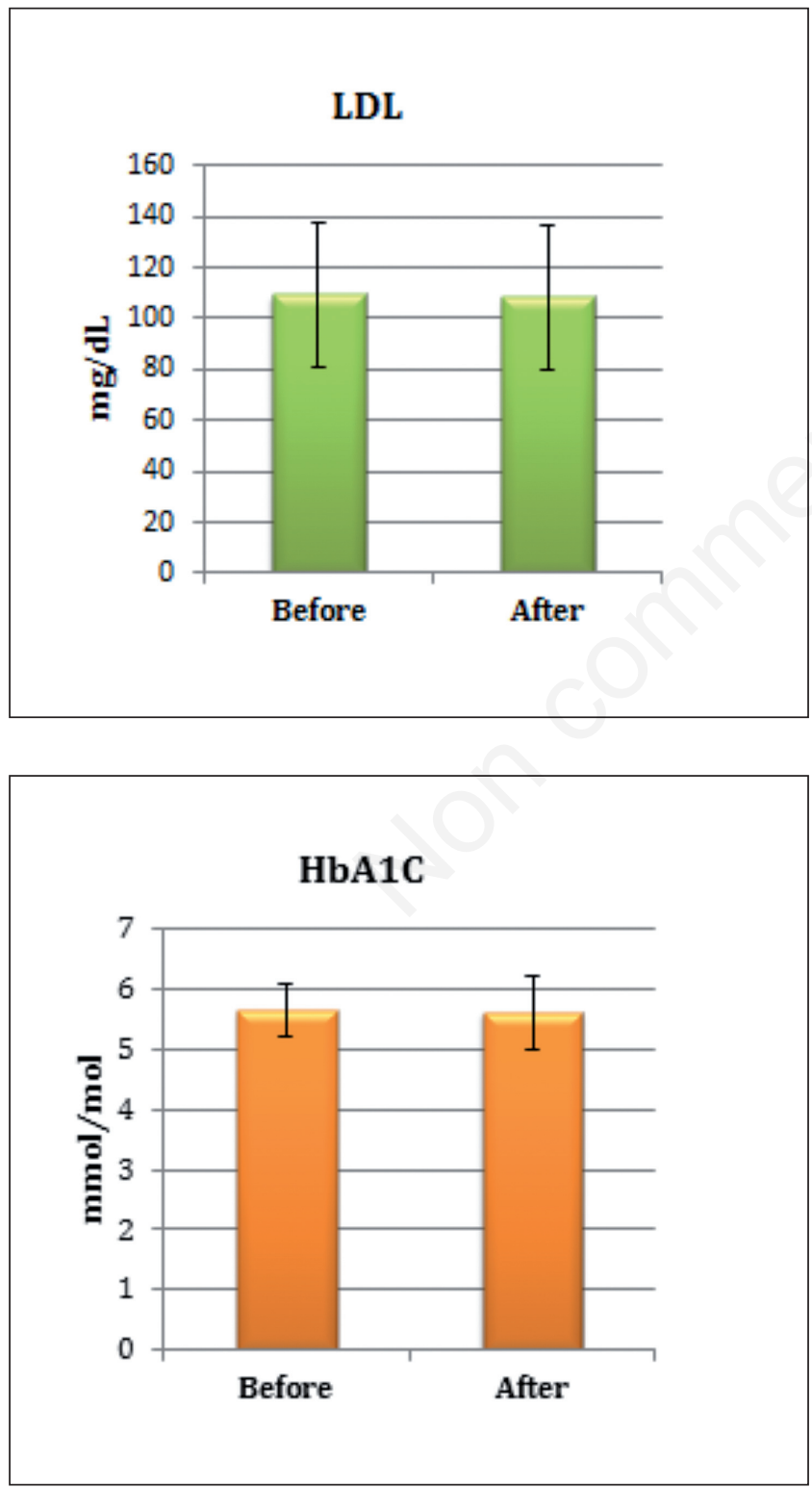

with treatment was confirmed by the significant effects on spirometry parameters and clinical improvement. The results of logistic regression analysis showed that there was not a significant relation between age, sex, FEV1, FVC and CRP with HOMA-IR after treatment except FEV1/FVC that this relation was statistical significant, but after adjusting for confounding variables the relation was not statistical significant (Table 7).

Table 5. The mean of beta cell function before and after intervention.

\begin{tabular}{lcc} 
& Mean \pm SD & p-value \\
Beta cell function B\% & $94.70 \pm 29.48$ & \\
Beta cell function A\% & $104.02 \pm 40.53$ & $0.127^{*}$ \\
\hline
\end{tabular}

${ }^{*} t$-test.
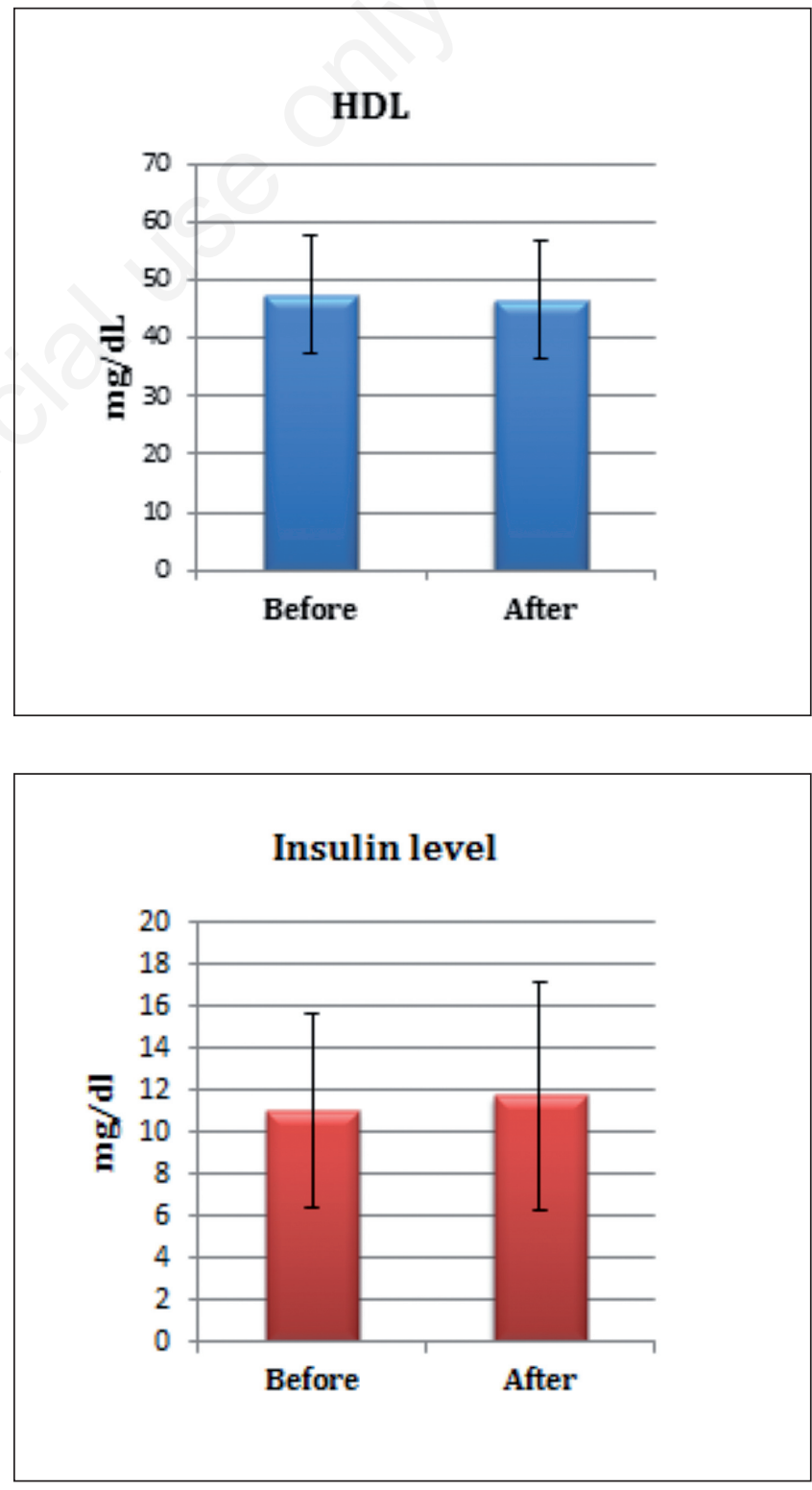

Figure 1 . The mean of LDL, HDL, HbA1C, and insulin level before and after treatment. 


\section{Discussion}

It has been clarified that a slight increase in insulin resistance may occur after ICS use in non-diabetic patients [9]. However, the effects of ICS on insulin sensitivity in these patients have hardly been investigated, and there are contradictory results on this issue [5]. The present study indicated that an average dose of ICS ( $320 \mu \mathrm{g}$ budesonide every 12 hours) for a period of 8 weeks creates a remarkable improvement in respiratory function and a significant reduction in patient's symptoms. Despite the satisfying outcomes of respiratory function in asthmatic patients, there is any change in insulin resistance outcome. This improvement in respiratory function is also indicative of patients' adherence to medication regiment.

Differences in individual insulin sensitivities can be explained by different factors such as age, gender, BMI, habits, activities, and severe infections. In the present study, we attempted to neutralize the confounders asking the patients to continue with their routine diet, habits, and daily activities during the treatment which resulted in a constant BMI.

In a large population-based cohort study of asthma and COPD patients, the results showed that the use of inhaled corticosteroids is associated with a significant $34 \%$ increase in the risk of incident diabetes especially in COPD patients. This risk increased with higher doses of inhaled corticosteroids such as $1000 \mu \mathrm{g}$ of fluticasone per day or equivalent [10]. In another cohort study that evaluated 1494 cases and 14,931 controls using density sampling. They did not observe a statistically significant increase in risk among users of high-dose beclomethasone compared to nonusers [11]. Slatore et al. evaluated in a cohort study the association of inhaled corticosteroid use with serum glucose concentration, they found there is no association between ICS and serum glucose after controlling for systemic corticosteroid use and other potential confounders [12].

Methods of measuring insulin resistance are insufficient and debatable. The amount of insulin and glucose in OGTT cannot be used to examine basic insulin sensitivity in diabetic patients whose fasting glucose level is over $160 \mathrm{mg} / \mathrm{dl}$ [5]. Few studies have investigated the effects of ICS on insulin sensitivity in asthmatic patients, and their results are controversial. The present study indicated that HOMA-IR, iHOMA2, beta cell function, insulin sensitivity, glucose and insulin level were not changed due to treatment with ICS in patients with asthma. Other studies reported similar results; low dose ICS (0.4 mg beclomethasone per day, equal to $400 \mu \mathrm{g}$ budesonide) was prescribed for 9 asthmatic patients who did not receive steroid for a period of 4 weeks, the results indicated that insulin and glucose levels did not change in 0GTT and hypothalamic-pituitary-adrenal (HPA) axis was not controlled [13]. The results of another study that was carried out on 9 asthmatic children indicated that the area under the curve of glucose (AUC glu) and the area ynder the curve of insulin (AUC ins) did not change with a $800 \mu \mathrm{gg} / \mathrm{m} / \mathrm{d}$ dose of inhaled budesonide for one month and $400 \mu \mathrm{g} / \mathrm{m} / \mathrm{d}$ for 5 months [14]. On the other hand, the results of a study conducted on 15 patients with severe asthma treated with $2 \mathrm{mg}$ followed by $1 \mathrm{mg}$ beclomethasone or $800 \mu \mathrm{g}$ budesonide for 3 months indicated that in spite of their asthma was well controlled, the results of examining the areas under the curve of glucose and insulin indicated that insulin sensitivity increased while HPA axis did not change [15]. The difference between the results of this study with other studies can be attributable to the limited number of the samples and a severe grade of asthma, longer periods and higher doses of ICS treatment.

Most previous studies have utilized AUC glu and AUC ins measurement in order to investigate insulin sensitivity. In the present study, HOMA-IR and IHOMA2 was utilized, which is a reliable scientific method for examining insulin sensitivity. In 2007, Canis et al. employed HOMA-IR to investigate insulin sensitivity in 12 patients with asthma and COPD; and there was no significant difference between the amount of HOMA-IR, glucose, insulin in OGTT before and after the treatment [5] The results of the present study also indicated that HOMA-IR index was not influenced by asthma treatment with ICS.

In a prospective study of 103,614 nurses over a period of 8 years, it was reported that BMI and diabetes were 1.9 times more among patients with COPD compared with those without it and the risk of diabetes development in asthmatic and non-asthmatic patients was not different [16].

The limitation of our study is low sample size, low duration of treatment, medium dose of ICS, exclusion of obese patients and of patients with a family history of diabetes mellitus. So we suggest a future study with a larger sample size and inclusion of all patients with different BMI in longer periods to evaluate the effect of ICS on insulin sensitivity.

Table 6. Comparison mean of iHOMA and beta cell function and insulin sensitivity before and after treatment according to BMI.

\begin{tabular}{|c|c|c|c|c|c|c|c|c|c|}
\hline & \multicolumn{2}{|c|}{$\begin{array}{c}\text { iHOMA2 } \\
\text { Mean } \pm \text { SD }\end{array}$} & \multirow[t]{2}{*}{ p-value } & \multicolumn{2}{|c|}{$\begin{array}{c}\text { Beta cell function \% } \\
\text { Mean } \pm \text { SD }\end{array}$} & \multirow[t]{2}{*}{ p-value } & \multicolumn{2}{|c|}{$\begin{array}{c}\text { Insulin sensitivity \% } \\
\text { Mean } \pm \text { SD }\end{array}$} & \multirow[t]{2}{*}{ p-value } \\
\hline & B & A & & B & A & & B & A & \\
\hline $\mathrm{BMI}<25$ & $1.55 \pm .69$ & $1.85 \pm .93$ & 0.213 & $89.35 \pm 30.02$ & $108.05 \pm 43.68$ & 0.058 & $75.84 \pm 29.94$ & $63.7364 \pm 24.20$ & 0.155 \\
\hline BMI $=25-30$ & $1.40 \pm .55$ & $1.34 \pm .44$ & 0 & $98.16 \pm 29.519$ & $101.4176 \pm 39.51$ & 0.682 & $87.96 \pm 54.67$ & $83.5824 \pm 33.84$ & 0.653 \\
\hline
\end{tabular}

BMI: Body mass index.

Table 7. Relationship between HOMA-IR and demographic variables after treatment.

\begin{tabular}{lcccc} 
Variables & Crude OR and 95\% CI & p-value & Adjusted OR and 95\% CI & p-value \\
Age & $0.980(0.920-1.044)$ & 0.524 & $0.929(0.834-1.034)$ & 0.175 \\
\hline Sex & $2.400(0.524-10.992)$ & 0.259 & $1.233(0.159-9.595)$ & 0.841 \\
\hline FEV1 & $1.048(0.971-1.130)$ & 0.228 & $1.253(0.989-1.586)$ & 0.062 \\
\hline FVC & $0.956(0.875-1.045)$ & 0.326 & $0.794(0.620-1.016)$ & 0.067 \\
\hline FEV1/FVC & $1.162(1.027-1.313)$ & $0.017 *$ & $1.034(0.870-1.230)$ & 0.703 \\
\hline CRP & $1.000(0.186-5.379)$ & 1.000 & $0.694(0.044-11.027)$ & 0.796
\end{tabular}

*Statistically significant. 


\section{Conclusions}

The results of the present study indicated that ICS usage leads to a remarkable increase in the amount of FEV1, FVC and FEV1/FVC, However, none of the parameters relating to insulin sensitivity changed significantly. Furthermore, we concluded there is no relationship between ICS use and insulin sensitivity in asthmatic patients.

\section{References}

1. Tavacol H, Rahimi Z, Cheraghi M, et al. A cross-sectional study of prevalence and risk factors for childhood asthma in Ahvaz city, Iran. Adv Dermatol Allergol 2015;32:268-73.

2. Gulcan E, Bulut I, Toker A, Gulcan A. Evaluation of glucose tolerance status in patients with asthma bronchiale. J Asthma 2009;46:207-9.

3. Tesse R, Schieck M, Kabesch M. Asthma and endocrine disorders: shared mechanisms and genetic pleiotropy. Mol Cell Endocrinol 2011;333:103-11.

4. Louis M, Punjabi NM. Effects of acute intermittent hypoxia on glucose metabolism in awake healthy volunteers. J Appl Physiol 2009;106:1538-44.

5. Canis R, Demirkok SS, Osar Z, et al. Effects of inhaled budesonide on insulin sensitivity in nondiabetic patients with asthma and chronic obstructive pulmonary disease. Adv Therapy 2007;24:560-70.

6. Faul JL, Wilson SR, Chu JW, et al. The effect of an inhaled corticosteroid on glucose control in type 2 diabetes. Clin Med Res 2009; 7:14-20.
7. Thuesen B, Husemoen L, Hersoug LG, et al. Insulin resistance as a predictor of incident asthma-like symptoms in adults. Clin Exp Allergy 2009;39:700-7.

8. Blackburn D, Hux J, Mamdani M. Quantification of the risk of corticosteroid-induced diabetes mellitus among the elderly. J Gen Inter Med 2002;17:717-20.

9. Rewers M, Zaccaro D, D’Agostino R, et al. Insulin sensitivity, insulinemia, and coronary artery disease: the Insulin Resistance Atherosclerosis Study. Diabetes Care 2004;27:781-7.

10. Suissa S, Kezouh A, Ernst P. Inhaled corticosteroids and the risks of diabetes onset and progression. Am J Med 2010;123:1001-6.

11. Dendukuri N, Blais L, LeLorier J. Inhaled corticosteroids and the risk of diabetes among the elderly. Br J Clin Pharmacol 2002;54:59-64.

12. Slatore CG, Bryson CL, Au DH. The association of inhaled corticosteroid use with serum glucose concentration in a large cohort. Am J Med 2009;122:472-8.

13. Yernault J-C, Leclercq R, Schandevyl W, et al. The endocrinometabolic effects of beclomethasone dipropionate in asthmatic patients. Chest 1977;71:698-702.

14. Turpeinen M, Sorva R, Juntunen-Backman K. Changes in carbohydrate and lipid metabolism in children with asthma inhaling budesonide. J Allergy Clin Immun 1991;88:384-9.

15. Kiviranta K, Turpeinen M. Effect of eight months of inhaled beclomethasone dipropionate and budesonide on carbohydrate metabolism in adults with asthma. Thorax 1993;48:974-8.

16. Rana JS, Mittleman MA, Sheikh J, et al. Chronic obstructive pulmonary disease, asthma, and risk of type 2 diabetes in women. Diabetes Care 2004;27:2478-84. 